\title{
Issues, Principles and Practice in the Improvement of Productive Skills
}

Miguel Fernandez (Chicago State University)

Jennifer Forth (University of Kansas)

Abby Franchitti (Kansas State University)

Peggy Allen Heidish (Carnegie Mellon University)

Heather Robertson (University of Southern California)

One area of research I am interested in is helping students improve production skills. This session description for a panel discussion at TESOL 2013 summarizes my interest in this area.

\section{Session Description}

While speaking and writing are substantially different in many ways, they both are used for the same purpose - to communicate. In many ways, writing is one of the most difficult skills, requiring a greater degree of accuracy. When speaking, any misunderstandings can be cleared up 'on the spot', whereas this is not possible with writing. Speaking, on the other hand, requires a greater degree of fluency as the speaker will rarely have time to think and plan an answer. Communication between people is a very complex and ever changing thing. But there are generalizations that we can make which have particular relevance for the teaching and learning of languages.

Speaking is considered by learners as one of the most difficult skills as it involves real-time processing which means that learners don't have much time to formulate what they want to say and how to say it. As a result students often avoid speaking and therefore never get the opportunity to build up confidence through practice. A further reason that students avoid speaking is that they are afraid to make mistakes because of poor pronunciation. This poor pronunciation often stems from previous learning experiences where there has been an emphasis on written accuracy with little chance to develop oral skills. Students may also, in many cases, have little opportunity to practice their English speaking outside of the classroom environment.

Written texts, on the other hand, have quite a number of differences that separate them from speaking. Not only are there differences in grammar (for example usage of contracted forms in speaking are often not applied in writing), and vocabulary (usually more formal in written English) there are also the added factors of spelling, handwriting, layout and punctuation. Despite these differences many of the same factors relevant to teaching speaking are also relevant to teaching writing.

The focus of my research interests and this academic session for TESOL is to present the issues and challenges involved in improving speaking and writing skills with Higher Education students, and evaluate different ways to address these. The session will help develop understanding of the skills of speaking, pronunciation and writing in the relevant teaching contexts, and will also address teacher confidence to teach these skills by demonstrating a range of practical classroom activities that support the development of productive skills. 


\section{Survey of Faculty, Administration and GTA Interests for 2013 The Complete Results}

\section{What is your current position at the AEC?}

\section{Response Response \\ Percent Count}

Faculty (e.g., pool lecturer, multi-term lecturer, permanent lecturer, language specialist)

\section{Administrator}

$6.0 \%$

GTA

$4.0 \%$

2. How many total years have you worked at the AEC?

\begin{tabular}{|c|c|c|}
\hline & $\begin{array}{c}\text { Response } \\
\text { Percent }\end{array}$ & $\begin{array}{c}\text { Response } \\
\text { Count }\end{array}$ \\
\hline $0-3$ years & $36.0 \%$ & 18 \\
\hline 4-7 years & $20.0 \%$ & 10 \\
\hline 8-11 years & $10.0 \%$ & 5 \\
\hline $12-15$ years & $10.0 \%$ & 5 \\
\hline $16-19$ years & $6.0 \%$ & 3 \\
\hline \multirow[t]{3}{*}{$20+$ years } & $18.0 \%$ & 9 \\
\hline & answered question & 50 \\
\hline & skipped question & 0 \\
\hline
\end{tabular}




\section{Have you held different positions within the AEC?}

\begin{tabular}{|c|c|c|}
\hline & $\begin{array}{c}\text { Response } \\
\text { Percent }\end{array}$ & $\begin{array}{c}\text { Response } \\
\text { Count }\end{array}$ \\
\hline Yes & $54.0 \%$ & 27 \\
\hline \multirow[t]{3}{*}{ No } & $46.0 \%$ & 23 \\
\hline & answered question & 50 \\
\hline & skipped question & 0 \\
\hline
\end{tabular}

\section{If so, for how many years have you held each position below?}

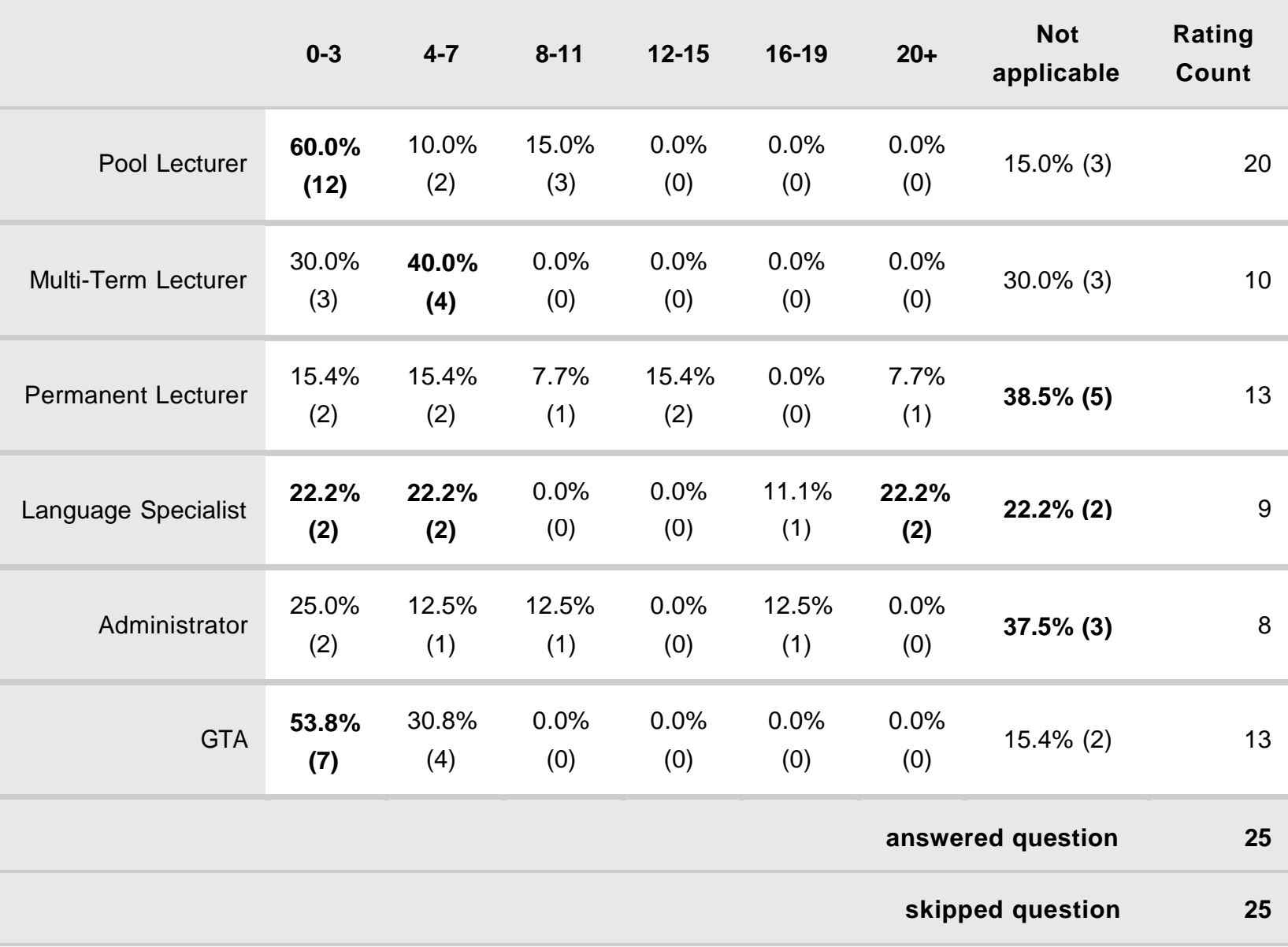


5. How many years have you been in the field of TESL/TEFL?

\begin{tabular}{r|rr} 
& $\begin{array}{r}\text { Response } \\
\text { Percent }\end{array}$ & $\begin{array}{c}\text { Response } \\
\text { Count }\end{array}$ \\
\hline $0-5$ & $14.3 \%$ & 7 \\
\hline $6-10$ & $18.4 \%$ & 9 \\
\hline $11-15$ & $10.2 \%$ & 5 \\
\hline $16-20$ & $10.2 \%$ & 5 \\
\hline $21+$ & answered question & 49 \\
\hline & skipped question & $\mathbf{2 3}$ \\
\hline
\end{tabular}

6. How many years of graduate school do you have beyond your (first) Master's degree?

\begin{tabular}{rrr} 
& $\begin{array}{r}\text { Response } \\
\text { Percent }\end{array}$ & $\begin{array}{c}\text { Response } \\
\text { Count }\end{array}$ \\
\hline $\mathbf{0 - 2}$ & $63.3 \%$ & $\mathbf{3 1}$ \\
\hline $3-6$ & $26.5 \%$ & 13 \\
\hline $7-10$ & $4.1 \%$ & 2 \\
\hline $10+$ & $6.1 \%$ & 3 \\
\hline & answered question & 49 \\
\hline & skipped question & $\mathbf{1}$ \\
\hline
\end{tabular}


7. Do you have an ABD?

\begin{tabular}{lrr} 
& & $\begin{array}{c}\text { Response Response } \\
\text { Percent } \\
\text { Count }\end{array}$ \\
Yes & $12.2 \%$ & 6 \\
\hline & answered question & 49 \\
& skipped question & 1
\end{tabular}

8. Do you have a Ph.D. or equivalent?

\begin{tabular}{lrrr} 
& $\begin{array}{c}\text { Response } \\
\text { Percent }\end{array}$ & $\begin{array}{c}\text { Response } \\
\text { Count }\end{array}$ \\
\hline Yes & $18.4 \%$ & 9 \\
\hline No & $\mathbf{8 1 . 6 \%}$ & $\mathbf{4 0}$
\end{tabular}

answered question

9. Did you study abroad?

\begin{tabular}{|c|c|c|}
\hline & $\begin{array}{c}\text { Response } \\
\text { Percent }\end{array}$ & $\begin{array}{c}\text { Response } \\
\text { Count }\end{array}$ \\
\hline Yes & $57.1 \%$ & 28 \\
\hline \multirow[t]{3}{*}{ No } & $42.9 \%$ & 21 \\
\hline & answered question & 49 \\
\hline & skipped question & 1 \\
\hline
\end{tabular}


10. For approximately how long did you study abroad?

\begin{tabular}{|c|c|c|}
\hline & $\begin{array}{c}\text { Response } \\
\text { Percent }\end{array}$ & $\begin{array}{c}\text { Response } \\
\text { Count }\end{array}$ \\
\hline 1-6 Weeks & $7.1 \%$ & 2 \\
\hline 1 Semester & $35.7 \%$ & 10 \\
\hline 1 Year & $32.1 \%$ & 9 \\
\hline \multirow[t]{3}{*}{ More than 1 Year } & $25.0 \%$ & 7 \\
\hline & answered question & 28 \\
\hline & skipped question & 22 \\
\hline
\end{tabular}

11. Have you ever worked abroad?

\begin{tabular}{|c|c|c|}
\hline & $\begin{array}{c}\text { Response } \\
\text { Percent }\end{array}$ & $\begin{array}{c}\text { Response } \\
\text { Count }\end{array}$ \\
\hline Yes & $71.4 \%$ & 35 \\
\hline \multirow[t]{3}{*}{ No } & $28.6 \%$ & 14 \\
\hline & answered question & 49 \\
\hline & skipped question & 1 \\
\hline
\end{tabular}

12. For approximately how long did you work abroad?

\begin{tabular}{rrr} 
& $\begin{array}{r}\text { Response } \\
\text { Percent }\end{array}$ & $\begin{array}{c}\text { Response } \\
\text { Count }\end{array}$ \\
\hline Less than 1 Year & $17.1 \%$ & 6 \\
\hline $1-2$ Years & $31.4 \%$ & 11 \\
\hline More than 2 Years & $51.4 \%$ & 18 \\
\hline & answered question & 35 \\
\hline & skipped question & 15 \\
\hline
\end{tabular}


13. Do you know a language other than English?

\begin{tabular}{|c|c|c|}
\hline & $\begin{array}{c}\text { Response } \\
\text { Percent }\end{array}$ & $\begin{array}{c}\text { Response } \\
\text { Count }\end{array}$ \\
\hline Yes & $85.7 \%$ & 42 \\
\hline \multirow[t]{3}{*}{ No } & $14.3 \%$ & 7 \\
\hline & answered question & 49 \\
\hline & skipped question & 1 \\
\hline
\end{tabular}

14. Answer the following questions about the language you know best other than English. On an AEC scale, comment on your ability in the other language. (If you know two or more additional languages equally well, please choose one language.) NR = Beyond AEC level 5. "Myability is roughly equivalent to":

\begin{tabular}{|c|c|c|c|c|c|c|c|}
\hline & 1 & 2 & 3 & 4 & 5 & NR & $\begin{array}{l}\text { Rating } \\
\text { Count }\end{array}$ \\
\hline Speaking/Listening Level & $7.1 \%(3)$ & $\begin{array}{c}23.8 \% \\
(10)\end{array}$ & $\begin{array}{c}26.2 \% \\
(11)\end{array}$ & $19.0 \%(8)$ & $14.3 \%(6)$ & $9.5 \%(4)$ & 42 \\
\hline Reading/Writing Level & $12.2 \%(5)$ & $22.0 \%(9)$ & $\begin{array}{c}31.7 \% \\
(13)\end{array}$ & $14.6 \%(6)$ & $12.2 \%(5)$ & $7.3 \%(3)$ & 41 \\
\hline Grammar for Communication Level & $7.3 \%(3)$ & $19.5 \%(8)$ & $\begin{array}{c}34.1 \% \\
(14)\end{array}$ & $19.5 \%(8)$ & $12.2 \%(5)$ & $7.3 \%(3)$ & 41 \\
\hline \multicolumn{7}{|c|}{ answered question } & 42 \\
\hline \multicolumn{7}{|c|}{ skipped question } & 8 \\
\hline
\end{tabular}




\section{General Professional Interests On a scale of 1-5, rate your professional interest in the following. One is the lowest and 5 is the highest. 'DK' refers to 'I don't know.'}

$\begin{array}{llllllll}1 & 2 & 3 & 4 & 5 & \text { DK } & \text { Rating } \\ \text { Count }\end{array}$

Teaching Methodologies or Approaches (e.g., communicative, content-based, skill-based, corpus-

based, task-based, immersion, CALL, etc.)

\begin{tabular}{r|ccc}
\hline Assessment & $0.0 \%(0)$ & $12.2 \%(6)$ & $\begin{array}{c}\mathbf{4 0 . 8 \%} \\
(20)\end{array}$ \\
\hline Curriculum Design & $2.1 \%(1)$ & $6.3 \%(3)$ & $\begin{array}{c}22.9 \% \\
(11)\end{array}$ \\
\hline Materials Writing & $8.3 \%(4)$ & $4.2 \%(2)$ & $10.4 \%(5)$ \\
\hline & & & \\
\hline
\end{tabular}

Intercultural Communication

Second Language Acquisition

Second Language Acquisition

Administration

English for Academic Purposes

Application of Theory to TESL/TEFL

(e.g., Cognitive Linguistics,

Systemic Functional Linguistics,

Academic literacies, Complex Systems theory, etc.)

Advancing the field (e.g., holding office in professional organization, presenting at professional conferences, publishing your work,

etc.)

$0.0 \%(0) \quad 8.2 \%(4) \quad 12.2 \%(6)$

$16.3 \%(8) \quad 28.6 \% \quad 22.4 \%$

(14) (11)

$2.0 \%(1) \quad 4.1 \%(2) \quad 14.3 \%(7)$

$16.3 \%(8)$

$22.4 \% \quad 20.4 \%$

(11) (10)

$6.3 \%(3) \quad 10.4 \%(5)$

$31.3 \%$
$(15)$

$\begin{array}{lcccccc} & & 22.4 \% & 24.5 \% & \mathbf{4 6 . 9} \% & 0.0 \%(0) & 49\end{array}$

$\begin{array}{lcc}(19) & \mathbf{3 8 . 8} \% & 2.0 \%(1)\end{array}$

49

$\begin{array}{lcccccc} & & 22.9 \% & 29.2 \% & 33.3 \% & 0.0 \%(0) & 48\end{array}$

$16.3 \%(8) \quad 14.3 \%(7) \quad 2.0 \%(1)$

49

$34.7 \%$

(17)

$42.9 \%$

(21)

$2.0 \%(1)$

49

$20.4 \%$

(10)

$18.4 \%(9) \quad 2.0 \%(1)$

49

$27.1 \%$

$22.9 \%$

$2.1 \%(1)$

48

answered question 


\section{1 \\ 23 \\ 4 \\ 5 \\ DK \\ Rating \\ Count}

\begin{tabular}{l|llccccc} 
Level 1 & $16.3 \%(8)$ & $12.2 \%(6)$ & $\mathbf{2 6 . 5 \%}$ & $18.4 \%(9)$ & $18.4 \%(9)$ & $8.2 \%(4)$ & 49
\end{tabular}

$\begin{array}{llllcccc}\text { Level } 2 & 14.3 \%(7) & 8.2 \%(4) & 18.4 \%(9) & 26.5 \% & \mathbf{2 8 . 6 \%} & 4.1 \%(2) & 49\end{array}$

\begin{tabular}{l|ccccccc} 
& & & & $30.6 \%$ & $\mathbf{3 2 . 7 \%}$ & $6.1 \%(3)$ & 49
\end{tabular}

\begin{tabular}{|c|c|c|c|c|c|c|}
\hline Level 4 & $4.2 \%(2)$ & $6.3 \%$ (3) & $12.5 \%(6)$ & $\begin{array}{c}29.2 \% \\
(14)\end{array}$ & $\begin{array}{c}43.8 \% \\
(21)\end{array}$ & $4.2 \%(2)$ \\
\hline
\end{tabular}

\begin{tabular}{|l|ccccccc}
\hline Level 5 (ESLP 110) & $10.6 \%(5)$ & $4.3 \%(2)$ & $19.1 \%(9)$ & $\begin{array}{c}\mathbf{3 6 . 2} \% \\
\mathbf{( 1 7 )}\end{array}$ & $\begin{array}{c}21.3 \% \\
(10)\end{array}$ & $8.5 \%(4)$ & 47
\end{tabular}

Special Studies (e.g., ESLP 122;

ESLP 126; ESLP 128)

$8.5 \%(4) \quad 8.5 \%(4) \quad 12.8 \%(6)$

$23.4 \%$

(11)

$38.3 \%$

(18)

$8.5 \%(4)$

47

Speaking/Listening $\quad 10$

$10.2 \%(5) \quad 16.3 \%(8)$

$22.4 \%$

(11)

$14.3 \%(7)$

$3<.1 \%$

$4.1 \%(2)$

49

Reading/Writing

$12.2 \%(6) \quad 4.1 \%(2) \quad 12.2 \%(6)$

$20.4 \%$

(10)

$49.0 \%$
$(24)$$\quad 2.0 \%(1)$

Grammar for Communication

$10.6 \%(5) \quad 6.4 \%(3) \quad 12.8 \%(6)$

$27.7 \%$

(13)

$40.4 \%$

(19)

$2.1 \%(1)$

47

Short-term Programs

$4.1 \%(2) \quad 2.0 \%(1) \quad 12.2 \%(6)$

$26.5 \%$

(13)

$42.9 \%$

$12.2 \%(6)$

49

The Graduate Writing Program

$28.6 \%$

(14)

$18.4 \%(9) \quad 14.3 \%(7)$

$16.3 \%$

(8)

$14.3 \%(7)$

$8.2 \%(4)$

49

answered question 


\section{Non-teaching Interests On a scale of 1-5, rate your professional interest in the following. One is the lowest and 5 is the highest. 'DK' stands for 'don't know.'}

\begin{tabular}{|c|c|c|c|c|c|c|c|}
\hline & 1 & 2 & 3 & 4 & 5 & DK & $\begin{array}{l}\text { Rating } \\
\text { Count }\end{array}$ \\
\hline $\begin{array}{r}\text { Being an AEC counselor/academic } \\
\text { advisor }\end{array}$ & $\begin{array}{c}33.3 \% \\
(16)\end{array}$ & $18.8 \%(9)$ & $\begin{array}{c}20.8 \% \\
(10)\end{array}$ & $8.3 \%(4)$ & $16.7 \%(8)$ & $2.1 \%(1)$ & 48 \\
\hline Grading SPEAK tests & $\begin{array}{c}31.3 \% \\
(15)\end{array}$ & $\begin{array}{c}22.9 \% \\
(11)\end{array}$ & $10.4 \%(5)$ & $18.8 \%(9)$ & $12.5 \%(6)$ & $4.2 \%(2)$ & 48 \\
\hline Working in LEO Lab & $\begin{array}{c}43.8 \% \\
(21)\end{array}$ & $\begin{array}{c}22.9 \% \\
(11)\end{array}$ & $12.5 \%(6)$ & $6.3 \%(3)$ & $8.3 \%(4)$ & $6.3 \%(3)$ & 48 \\
\hline Working at The Point & $8.3 \%(4)$ & $\begin{array}{c}20.8 \% \\
(10)\end{array}$ & $\begin{array}{c}33.3 \% \\
(16)\end{array}$ & $\begin{array}{c}20.8 \% \\
(10)\end{array}$ & $12.5 \%(6)$ & $4.2 \%(2)$ & 48 \\
\hline Writing grants & $\begin{array}{c}24.5 \% \\
(12)\end{array}$ & $\begin{array}{l}22.4 \% \\
(11)\end{array}$ & $14.3 \%(7)$ & $14.3 \%(7)$ & $14.3 \%(7)$ & $10.2 \%(5)$ & 49 \\
\hline Being a coordinator of a class & $12.5 \%(6)$ & $6.3 \%(3)$ & $\begin{array}{l}20.8 \% \\
(10)\end{array}$ & $\begin{array}{c}22.9 \% \\
(11)\end{array}$ & $\begin{array}{c}33.3 \% \\
(16)\end{array}$ & $4.2 \%(2)$ & 48 \\
\hline Participating on committees & $4.3 \%(2)$ & $10.9 \%(5)$ & $\begin{array}{l}23.9 \% \\
(11)\end{array}$ & $\begin{array}{c}30.4 \% \\
(14)\end{array}$ & $\begin{array}{l}26.1 \% \\
(12)\end{array}$ & $4.3 \%(2)$ & 46 \\
\hline $\begin{array}{r}\text { Helping others with AEC technology } \\
\text { issues }\end{array}$ & $\begin{array}{c}25.0 \% \\
(12)\end{array}$ & $14.6 \%(7)$ & $\begin{array}{c}20.8 \% \\
(10)\end{array}$ & $\begin{array}{c}27.1 \% \\
(13)\end{array}$ & $10.4 \%(5)$ & $2.1 \%(1)$ & 48 \\
\hline \multirow[t]{2}{*}{$\begin{array}{r}\text { Offering in-house workshops, } \\
\text { presentations; facilitating brownbag } \\
\text { discussions, etc. }\end{array}$} & $2.0 \%(1)$ & $6.1 \%(3)$ & $\begin{array}{c}32.7 \% \\
(16)\end{array}$ & $\begin{array}{l}22.4 \% \\
(11)\end{array}$ & $\begin{array}{c}32.7 \% \\
(16)\end{array}$ & $4.1 \%(2)$ & 49 \\
\hline & $6.3 \%(3)$ & $4.2 \%(2)$ & $\begin{array}{c}25.0 \% \\
(12)\end{array}$ & $\begin{array}{c}37.5 \% \\
(18)\end{array}$ & $\begin{array}{c}20.8 \% \\
(10)\end{array}$ & $6.3 \%(3)$ & 48 \\
\hline Interacting with visiting scholars & $0.0 \%(0)$ & $8.3 \%(4)$ & $12.5 \%(6)$ & $\begin{array}{c}35.4 \% \\
(17)\end{array}$ & $\begin{array}{c}39.6 \% \\
(19)\end{array}$ & $4.2 \%(2)$ & 48 \\
\hline $\begin{array}{r}\text { Participating in a practicum with a } \\
\text { graduate student }\end{array}$ & $8.3 \%(4)$ & $10.4 \%(5)$ & $18.8 \%(9)$ & $\begin{array}{c}39.6 \% \\
(19)\end{array}$ & $18.8 \%(9)$ & $4.2 \%(2)$ & 48 \\
\hline $\begin{array}{l}\text { Participating in AEC out-of-class } \\
\text { activities (e.g., conversation } \\
\text { groups, trips, events, etc.) }\end{array}$ & $10.4 \%(5)$ & $\begin{array}{c}29.2 \% \\
(14)\end{array}$ & $\begin{array}{c}31.3 \% \\
(15)\end{array}$ & $14.6 \%(7)$ & $12.5 \%(6)$ & $2.1 \%(1)$ & 48 \\
\hline
\end{tabular}




\begin{tabular}{|c|c|c|c|c|c|c|c|}
\hline Observing colleagues/other classes & $0.0 \%(0)$ & $10.2 \%(5)$ & $14.3 \%(7)$ & $\begin{array}{c}40.8 \% \\
(20)\end{array}$ & $\begin{array}{c}30.6 \% \\
(15)\end{array}$ & $4.1 \%(2)$ & 49 \\
\hline \multicolumn{8}{|l|}{ Curriculum Review/Revision } \\
\hline Materials Development & $2.1 \%(1)$ & $6.4 \%(3)$ & $14.9 \%(7)$ & $\begin{array}{l}27.7 \% \\
(13)\end{array}$ & $\begin{array}{c}44.7 \% \\
(21)\end{array}$ & $4.3 \%(2)$ & 47 \\
\hline $\begin{array}{r}\text { Assessment (e.g., improving the } \\
\text { Proficiency Test) }\end{array}$ & $8.2 \%(4)$ & $10.2 \%(5)$ & $\begin{array}{l}24.5 \% \\
(12)\end{array}$ & $\begin{array}{l}26.5 \% \\
(13)\end{array}$ & $\begin{array}{c}28.6 \% \\
(14)\end{array}$ & $2.0 \%(1)$ & 49 \\
\hline $\begin{array}{r}\text { AEC Mission Development and } \\
\text { Review }\end{array}$ & $10.6 \%(5)$ & $\begin{array}{l}27.7 \% \\
(13)\end{array}$ & $\begin{array}{l}29.8 \% \\
(14)\end{array}$ & $10.6 \%(5)$ & $10.6 \%(5)$ & $10.6 \%(5)$ & 47 \\
\hline Decision-making Processes & $8.2 \%(4)$ & $\begin{array}{c}20.4 \% \\
(10)\end{array}$ & $18.4 \%(9)$ & $\begin{array}{c}20.4 \% \\
(10)\end{array}$ & $\begin{array}{c}22.4 \% \\
(11)\end{array}$ & $10.2 \%(5)$ & 49 \\
\hline Strategic planning & $10.4 \%(5)$ & $14.6 \%(7)$ & $18.8 \%(9)$ & $18.8 \%(9)$ & $\begin{array}{c}27.1 \% \\
(13)\end{array}$ & $10.4 \%(5)$ & 48 \\
\hline \multirow[t]{3}{*}{ New student orientation } & $8.5 \%(4)$ & $\begin{array}{c}21.3 \% \\
(10)\end{array}$ & $\begin{array}{c}27.7 \% \\
(13)\end{array}$ & $19.1 \%(9)$ & $17.0 \%(8)$ & $6.4 \%(3)$ & 47 \\
\hline & & & & & \multicolumn{2}{|c|}{ answered question } & 49 \\
\hline & & & & & \multicolumn{2}{|c|}{ skipped question } & 1 \\
\hline
\end{tabular}

